\title{
Origin and Evolution of the Moon's Procellarum KREEP Terrane
}

\author{
A White Paper submitted to the 2023 \\ Planetary Science Decadal Survey, July 15, 2020
}

Authorship

Brad Jolliff, Washington University in St. Louis, bjolliff@wustl.edu

Mark Robinson, Arizona State University, robinson@ser.asu.edu

Srinidhi Ravi, Arizona State University, sravi13@asu.edu

Co-signatories on the last page

The Procellarum KREEP Terrane on the Moon's nearside is a unique province that produced volcanism over an extended period of the Moon's history. Thermal evolution of the lunar mantle in this region may have been driven by a high concentration of radiogenic heat-producing elements. This region is key to unlocking the thermal evolution of the Moon and may have implications for our understanding of extensive volcanism on other inner Solar System bodies such as Mercury and Mars. Major volcanic features of this terrane could be explored by a long-distance rover in a New Frontiers class mission to answer questions about how this planetary asymmetry formed, why volcanism was so voluminous in this region, and how it persisted for nearly 4 billion years.

Introduction. The Moon displays a fundamental asymmetry that has been evident since the first images of the farside revealed an ancient, mountainous, heavily cratered feldspathic highlands but very little of the low-lying, dark basaltic plains that are prominent on the nearside, especially the western regions that incorporate Oceanus Procellarum, Imbrium, and several other nearside impact basins. Apollo orbital remote sensing revealed the first glimpses of compositional variations that corresponded to the hemispheric asymmetry, but only in narrow, near-equatorial orbital swaths. In 1994, the Clementine mission provided the first global multispectral mapping of the Moon and revealed for the first time the extent of the compositional asymmetry, which clearly corresponded to the asymmetric distribution of nearside vs. farside mare basalts. A global map of $\mathrm{FeO}$ derived from the UV-VIS multispectral data showed the FeO-rich volcanic regions of the nearside vs. the FeO-poor feldspathic highlands of the farside (Lucey et al., 1995). In the year of first results from the Lunar Prospector mission, Haskin (1998) interpreted the orbital gamma-ray data (Metzger et al., 1977) as representing an oval region of enrichment of the element thorium (Th), which is readily detected from orbit, owing to strong gamma-ray emissions from radioactive decay. Haskin predicted the possibility that the surface Th distribution arose largely from ejecta deposits of the Imbrium impact basin, which apparently excavated Th-rich material (Th is one of the elements concentrated in KREEP, a geochemical and rock component found in Apollo samples that are rich in K, REE, P, and other incompatible trace elements), but other large basins apparently did not excavate this material. Haskin surmised that this extraordinary concentration of KREEP materials would have great implications for the nature of the Moon's igneous differentiation and even for the nature of the proposed "cataclysm" or late-heavy bombardment of the Moon. 
The Lunar Prospector mission (1997-1998) then completed the global mapping of elements detected by orbital gamma-ray spectroscopy, including Th (e.g., Lawrence et al., 1998, 1999). The results showed strikingly what Haskin had predicted, namely an enormous oval region of Th enrichment in the Procellarum-Imbrium region. If the crustal rocks in this region were similarly enriched in Th, then a significant portion of the Moon's Th content must have been sequestered there. Just as important, however, was the observation that the enormous South Pole-Aitken basin on the southern farside was not similarly enriched, meaning that the KREEP materials were not globally distributed at depth. The Moon indeed was shown to have a profound compositional asymmetry that must have formed during its early differentiation. The significance of the Th enrichment, which appeared to pervade even the basalts of the Oceanus Procellarum region, led researchers to refer to the region as the "Procellarum KREEP Terrane" or PKT (Jolliff et al., 2000; Wieczorek and Phillips, 2000; Haskin et al., 2000) (Figure 1). Use of the geologic term "terrane" instead of "terrain" was intended to recognize that, because of its unique compositional character, it followed a different petrologic and thermal evolution, and thus, geologic history, than other parts of the Moon. Its petrologic evolution would be shown to include nearly 4 billion years (Byr) of magmatic history.

Unique volcanic history and prolonged magmatic activity. A defining characteristic of the Procellarum KREEP Terrane is its unique volcanic history. Outside of this terrane, the majority of mare basalts erupted within large impact basins where the low-density upper crust has been substantially thinned. In contrast to this behavior, Oceanus Procellarum represents the largest contiguous expanse of mare basalts, even though this region possesses a relatively thick crust and is not in an obvious basin setting (Wieczorek et al. 2001). While the PKT encompasses only about $16 \%$ of the surface area of the Moon, more than $60 \%$ of the mare basalts by area are located there. Furthermore, even though only $\sim 20 \%$ of the maria have $\mathrm{TiO}_{2}>5 \mathrm{wt} \%$, more than half of the basalts within the PKT have $\mathrm{TiO}_{2}$ concentrations that are $5 \mathrm{wt} \%$ or greater (Haskin et al., 2000).

The prolonged magmatic activity in the PKT is not predicted by general thermal evolution models of the Moon. After the initial differentiation of the Moon by solidification from the magma ocean of mafic cumulates to form the mantle and feldspathic cumulates to form the crust 4.4-4.5 Byr ago, large impactors and magmatic activity continued on the Moon until about 3.9 Byr ago when internal heating caused by radioactive $\mathrm{K}, \mathrm{U}$, and Th led to partial melting of the cumulate mantle and intrusion of those melts into the crust and extrusion onto the surface as mare basalts. The main active period of mare basaltic volcanism was 3.9 to 3.0 Byr, known from ages of basalts collected by Apollo missions. Using the basalt ages and radiometrically determined ages for several prominent craters on the Moon, including Tycho, Copernicus, and smaller craters at the landing sites, the lunar cratering chronology was developed (e.g., Neukum et al., 1975; Hartman et al., 1981). With the calibrated cratering curve, basalt ages were determined for many different regions of basalts, including the Oceanus Procellarum basalts (Hiesinger et al., 2000, 2003, 2011). While most of the basalts do indeed fall into the 3.0 to 3.9 Byr age range, some extend to younger ages, as young as 1.0 Byr (Stadermann et al., 2018). The younger basalts all occur within the PKT. If some of the radiogenic KREEP-rich material existed at depth within the mantle, the enhanced radioactive heating could possibly account for the extended production of basalt, even while other areas of the Moon cooled sufficiently to form a thick lithosphere. Thickening of the lithosphere coupled with increasing depth of partial melting over time would impede further volcanic activity elsewhere on the Moon, but not in the mantle beneath the PKT. And so goes the hypothesis for extended volcanic activity within the PKT. But is this really what happened? 


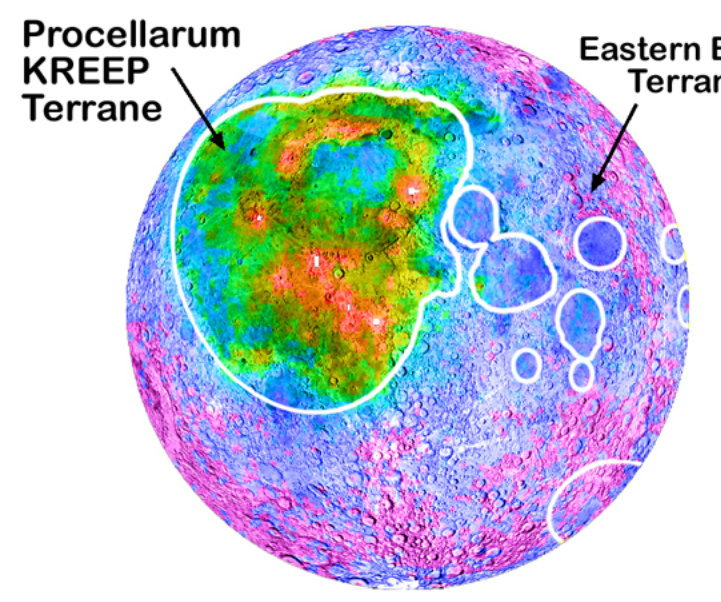

Near Side

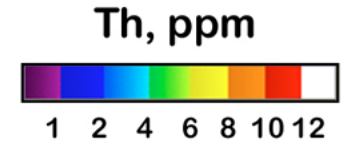

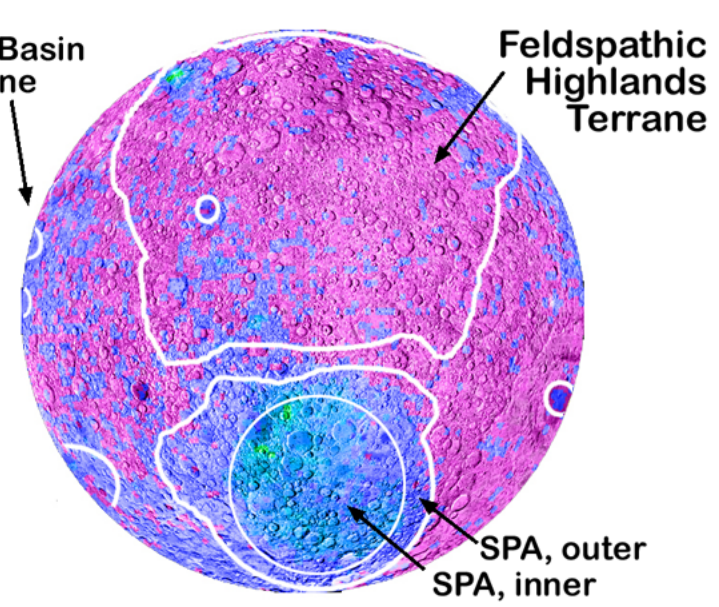

Far Side

Th concentrations from Lunar
Prospector data, calibrated to
landing site soils (Gillis et al., 2000)

Figure 1. Thorium map of the Moon outlining the area of the Procellarum KREEP Terrane on left, on the Moon's Earth-facing side, after Jolliff et al., 2000.

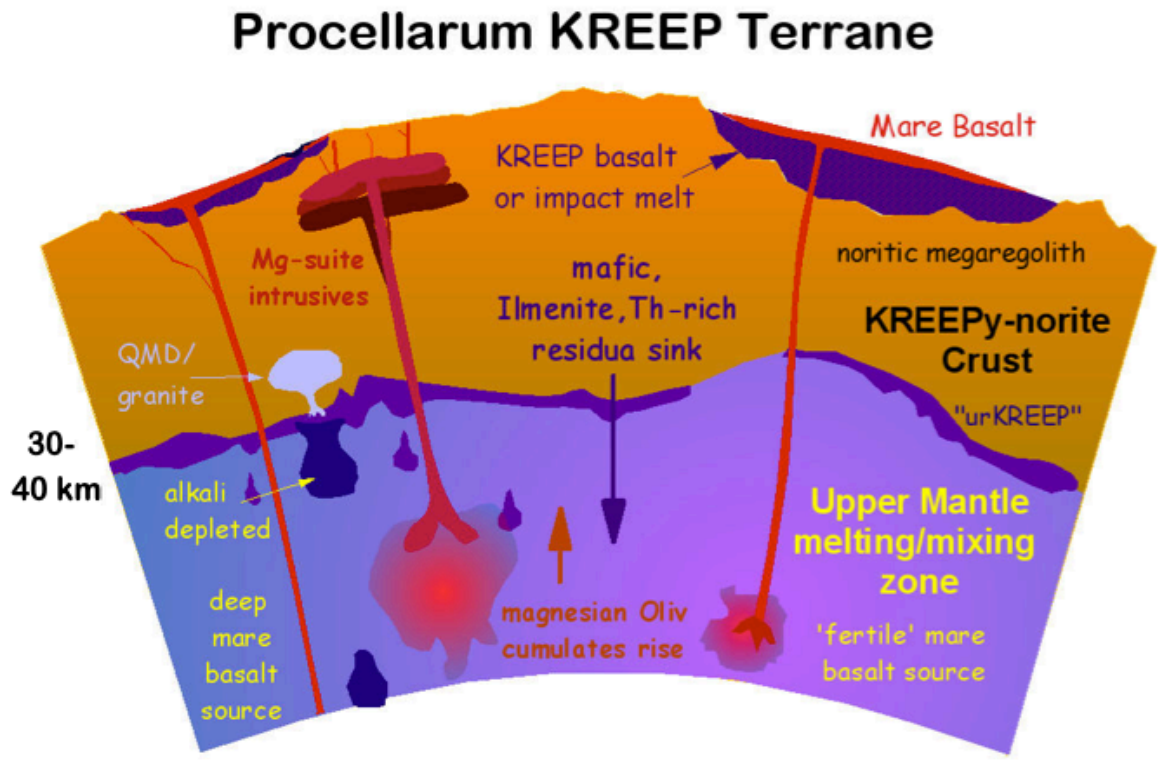

Figure 2. Schematic cross section illustrating some of the magmatic and volcanic processes that may have occurred in the Procellarum KREEP Terrane. After Jolliff et al., 2000. 
Subdued topography and viscous relaxation within the PKT. Despite that there is no firm evidence for structures such as basin rings to support the existence of an ancient, very large Procellarum impact basin, topography in the region is low (mean elevation below the lunar mean) and subdued (Haskin et al., 2000). Supporting the hypothesis that the PKT possesses a high abundance of heat-producing elements throughout its crustal section and possibly in the mantle beneath, heat-flow at Apollo 15, within the PKT, was found to be greater than heat flow at Apollo 17 (Langseth et al., 1976). Although the proximity of these two sites to the boundary of the PKT with the Feldspathic Highlands Terrane (FHT) renders these results ambiguous, other evidence suggests that the crust was hotter than typical within this region, causing impact-induced topography to relax viscously and faster than in the surrounding highlands. The rate of viscous relaxation depends on temperature. Since the Imbrium basin lies within the confines of the PKT, and Serenitatis is just on its border, a higher than typical crustal temperature at these locations could result from a higher abundance of radioactive elements. Perhaps the apparent shallow structure of these basins resulted from infilling by lavas with a density similar to that of the lower crust. KREEP basalt has a lower density than mare basalt and a composition similar to the mafic impact-melt breccias excavated by Imbrium and Serenitatis, which is consistent with KREEP basalt or an intrusive equivalent being a major chemical component within the crust of the PKT.

The isostatic state of large impact basins also supports higher crustal temperatures in the PKT. Some of the large impact basins appear to be super-isostatic, that is, the crust-mantle interface has been uplifted above the isostatic level. However, those basins that formed within or near the boundary of the PKT (Imbrium, Serenitatis, Grimaldi, and Humorum) appear to have been in an isostatic state prior to the emplacement of their mare fill (Wieczorek and Phillips 1999), consistent with enhanced viscous relaxation within the PKT.

Evidence of production of silicic volcanics by basaltic underplating at Aristarchus. One of the highest concentrations of thorium anywhere on the Moon is seen in the region surrounding Aristarchus crater. LRO Diviner data confirmed that the target region and much of the crater ejecta are silica-rich, suggesting that the Aristarchus impact target was a silicic volcanic or intrusive complex (Glotch et al., 2010). Near Aristarchus and well within the PKT are two other locations of prominent silicic volcanics, the Gruithuisen domes and the Mairan domes. The generation of silicic rocks on the Moon remains enigmatic (Valencia et al., 2020); however, recent observations of potential silicic volcanic materials on the Aristarchus Plateau (Zanetti et al., 2017) coupled with experimental results (Gullikson et al., 2016) support an origin by basaltic underplating and partial melting of fertile crust to produce silicic magma, suggested by Hagerty et al. (2006). Owing to the unique character of the PKT, this process was likely confined to this region, but all of the various aspects of these hypotheses need to be proven with on-surface, in-situ analysis.

\section{Outstanding questions}

Despite many orbital missions by multiple agencies in the 2000s and beyond, and years of analysis of high-quality remote sensing data, many questions remain regarding the characteristics and origin of the Procellarum KREEP Terrane. Although Lunar Prospector demonstrated that the PKT has a high surface abundance of incompatible elements, the gamma-ray and neutron spectrometers only sense the upper meter of the regolith. A major question is whether the KREEP component within this terrane is solely surficial (as in a veneer of KREEP-rich mare basalts and Imbrium 
ejecta), or whether it extends deep into the underlying crust and/or mantle. In either case, a compelling hypothesis is that the PKT surface composition and the extent of volcanism are genetically related. Impact-melt breccia excavated by Imbrium reflect a deep target region of the crust and possibly upper mantle rich in KREEP components and practically devoid of ferroan anorthositic rocks thought to make up a large portion of the primary crust outside of the PKT.

Some of the key scientific questions are as follows*:

- Are the basalts of Oceanus Procellarum themselves enriched in thorium and other heatproducing elements compared to other basalts sampled by Apollo, Luna, and lunar meteorites?

- Alternatively, is the apparent Th enrichment merely the result of vertical mixing with an underlying KREEP-rich substrate?

- Is there any correspondence between the ages of basalt units and their compositions?

- What factors led to the very different expressions of volcanic morphologies such as shield volcanos, domes and cones, pyroclastic deposits, and a new type of basaltic landform known as irregular mare patches (Braden et al., 2014)?

- What were the effects of impact craters such as Aristarchus that spewed Th-rich ejecta over large portions of the youngest basalts?

- What was the nature of the highly enriched rock that Aristarchus crater struck?

- What is the petrologic relationship between the basaltic rocks and the enriched crustal materials, if any?

- How and why did volcanism persist in this region over such a long span of time?

- How much of the distribution of KREEP-rich material was caused by the Imbrium basin impact?

- What caused the great concentration of KREEP in this region of the Moon?

- Was Oceanus Procellarum caused by a very ancient and large impact basin that formed while the crust was still too hot to retain structures commonly associated with such basins?

*Additional discussion of the Procellarum KREEP Terrane can be found in Wieczorek et al., 2006.

Origin of the Procellarum KREEP Terrane. The enrichment of incompatible elements and the unique volcanic history of the Procellarum KREEP Terrane bear strongly on the origin and evolution of this province. In particular, the observation that KREEP-rich materials are only abundant within this region of the Moon suggests that the final melt of the magma ocean was ultimately concentrated in this region. The process by which this accumulation might have occurred, however, is not clear. One hypothesis is that the crust in this region was thinner than typical, perhaps because of an early and now unrecognizable Procellarum impact event. Hydrostatic forces would then have caused a KREEP-rich magma sandwiched between the crust and mantle to accumulate beneath this region. Alternatively, the last remaining dregs of the lunar magma ocean may have accumulated above a degree-1 downwelling of dense ilmenite cumulates (Parmentier et al., 2002).

The high abundance of radioactive elements in the Procellarum KREEP Terrane also appears to be related to the voluminous and protracted volcanic history of this province. Wieczorek and 
Phillips (2000) showed that the inferred heat production of this province was likely more than sufficient to melt the underlying mantle over the duration of observed mare volcanism. Similarly, Parmentier et al. (2002) showed that if ilmenite cumulates of the late-stage magma ocean sank beneath the PKT, carrying with them a significant quantity of incompatible elements, melting of the mantle beneath this region would naturally occur as well. If and when such mantle overturn occurred and how deep the ilmenite cumulates may have sunk are not known but may be inferred from compositions coupled with ages of the volcanic products of the PKT.

Conclusion: Numerous characteristics of the Procellarum KREEP Terrane set it apart as a unique province on the Moon. However, such extensive volcanic provinces occur on other planets such as the northern plains of Mercury, and large volcanic provinces on Mars. What is the relationship between early planetary differentiation and the volcanic production of a differentiated body? How do planetary-scale asymmetries form and then affect the thermal evolution of planets? These are the sorts of questions that can be addressed by further exploration of the Procellarum KREEP Terrane. Furthermore, with a long-lived and long-distance capable rover, in-situ investigations of different volcanic manifestations within the PKT could potentially be explored with a New Frontiers Class mission. Perhaps the next decade will see the implementation of such a nextgeneration rover, and the Moon's Procellarum KREEP Terrane is the best location on the Moon for such an endeavor.

\section{References}

Braden, S. E., J. D. Stopar, M. S. Robinson, S. J. Lawrence, C. H. v. d. Bogert, H. Hiesinger (2014) Evidence for basaltic volcanism on the Moon within the past 100 million years. Nature Geoscience 7, 787-791.

Glotch, T. D., et al. (2010) Identification of highly silicic features on the Moon. Science 329, 1510-1513.

Gullikson, A. L., J. J. Hagerty, M. R. Reid, J. F. Rapp, D. S. Draper (2016) Silicic lunar volcanism: Testing the crustal melting model. Amer. Mineral. 101, 2312-2321.

Hagerty, J. J., et al. (2006) Refined thorium abundances for lunar red spots: Implications for evolved, nonmare volcanism on the Moon. J. Geophys. Res. 111, JE002592.

Hartmann, W. K., et al. (1981) Chronology of planetary volcanism by comparative studies of planetary craters, Basaltic Volcanism on the Terrestrial Planets. Pergamon Press, Elmsford, NY, pp. 1050-1127.

Haskin, L. A. (1998) The Imbrium impact event and the thorium distribution at the lunar highlands surface. J. Geophys. Res. 103, 1679-1689.

Haskin, L. A., J. J. Gillis, R. L. Korotev, B. L. Jolliff (2000) The materials of the Lunar Procellarum KREEP Terrane: A synthesis of data from geomorphological mapping, remote sensing, and sample analysis. $J$. Geophys. Res. 105, 20,403-420,416.

Hiesinger, H., R. Jaumann, G. Neukum (2000) Ages of mare basalts on the lunar nearside. J. Geophys. Res. 105, 29,239-229-275.

Hiesinger, H., J. W. Head, III, U. Wolf, R. Jaumann, G. Neukum (2003) Ages and stratigraphy of mare basalts in Oceanus Procellarum, Mare Nubium, Mare Cognitum, and Mare Insularum. J. Geophys. Res. 108, JE001985.

Hiesinger, H., J. W. I. Head, U. Wolf, R. Jaumann, G. Neukum (2011) Ages and stratigraphy of lunar mare basalts: A synthesis GSA Special Paper 477, 1-51.

Jolliff, B. L., J. J. Gillis, L. Haskin, R. L. Korotev, M. A. Wieczorek (2000) Major lunar crustal terranes: Surface expressions and crust-mantle origins. J. Geophys. Res. 105, 4197-4216. 
Langseth, M. G., S. J. Keihm, K. Peters (1976) Revised lunar heat-flow values. Proc. Lunar Sci. Conf. 7th, 3143-3171.

Lawrence, D. J., et al. (1998) Global elemental maps of the Moon: The Lunar Prospector gamma-ray spectrometer. Science 281, 1484-1489.

Lawrence, D. J., et al. (1999) High resolution measurements of absolute thorium abundance on the lunar surface. Geophys. Res. Lett. 26, 2681-2683.

Lawrence, D. J., et al. (2000) Thorium abundances on the lunar surface. $J$. Geophys. Res. 105, 20307-20331.

Lawrence, D. J., et al. (2007) Global spatial deconvolution of Lunar Prospector Th abundances. Geophys. Res. Lett. 34, L03201.

Lucey, P. G., G. J. Taylor, E. Malaret (1995) Abundance and distribution of iron on the Moon. Science 268, 1150-1153.

Metzger, A. E., et al. (1977) Thorium concentrations in the lunar surface, 1, Regional values and crustal content. Proc. Lunar Sci. Conf. 8th, 949-999.

Neukum, G., B. König, H. Fechting, D. Storzer (1975) Cratering in the Earth-moon system: Consequences for age determination by crater counting. Proc Lunar Science Conf 6, 2597-2620.

Parmentier, E. M., S. Zhong, M. T. Zuber (2002) Gravitational differentiation due to initial chemical stratification: Origin of lunar asymmetry by the creep of dense
KREEP? Earth Planet. Sci. Lett. 201, 473480.

Stadermann, A. C., M. R. Zanetti, B. L. Jolliff, H. Hiesinger, C. H. van der Bogert, C. W. Hamilton (2018) The age of lunar mare basalts south of the Aristarchus Plateau and effects of secondary craters formed by the Aristarchus event. Icarus 309, 45-60.

Valencia S. N., et al. (2020) Silicic Volcanics on the Moon. White Paper submitted for the Planetary Science Decadal Survey.

Wieczorek, M. A., R. J. Phillips (1999) Lunar multiring basins and the cratering process. Icarus 139, 246-259.

Wieczorek, M. A., R. J. Phillips (2000) The Procellarum KREEP terrane: Implications for mare volcanism and lunar evolution. $J$. Geophys. Res. 105, 20417-20430.

Wieczorek, M. A., M. T. Zuber, R. J. Phillips (2001) The role of magma buoyancy on the eruption of lunar basalts. Earth Planet. Sci. Lett. 185, 71-83.

Wieczorek, M. A., and many others (2006) The constitution and structure of the lunar interior (section 5.1) in New Views of the Moon, Reviews in Mineralogy and Geochemistry 60. Mineralogical Society of America, Washington, DC., pp. 221-364.

Zanetti, M., B. L. Jolliff, K. Shirley, T. D. Glotch, J. J. Hagerty, A. L. Gullikson (2017) The Aristarchus Plateau large igneous province: The case for bi-modal volcanism. Lunar Planet. Sci. 48, \#2320.

\section{$\underline{\text { Signatories }}$}

Daniel Moriarty, NASA Goddard Space Flight Center, daniel.p.moriarty@nasa.gov

James Tuttle Keane, Caltech/JPL, jkeane@caltech.edu

Matt Siegler, Planetary Science Institute, msiegler@psi.edu

Wajiha Iqbal, Westfalishce Wilhelms Universität, Iqbalw@uni-muenster.de

Kerri Donaldson Hanna, University of Central Florida, Kerri.DonaldsonHanna@ucf.edu

Emerson Speyerer, Arizona State University, espeyerer@ser.asu.edu

Eamonn Needham, Arizona State University, eneedha1@asu.edu

Ross Potter, Brown University, ross_potter@brown.edu

Nick Dygert, University of Tennessee, Knoxville,ndygert1@utk.edu

Devanshu Jha, MVJ College of Engineering, devanshu.jha7@gmail.com 